\title{
Local Measurement of Non-Classical Ion Heating During Magnetic Reconnection
}

\author{
S. C. Hsu, G. Fiksel,* T. A. Carter, H. Ji, R. M. Kulsrud, and M. Yamada \\ Princeton Plasma Physics Laboratory, P. O. Box 451, Princeton, NJ 08543
}

(November 11, 1999)

\begin{abstract}
Local ion temperature is measured directly in the well-characterized reconnection layer of a laboratory plasma. These measurements demonstrate definitively that ions are heated due to reconnection and that more than half of the reconnected field energy is converted to ion kinetic energy. Neither classical Ohmic dissipation nor thermalization of energetic flows is sufficent to account for the energy converted, suggesting the importance of non-classical dissipation mechanisms such as wave-particle interactions.
\end{abstract}

PACS numbers: 52.30.Jb, 96.60.Rd, 94.30.Lr

Typeset using REVTEX 
Magnetic reconnection $[1,2]$ has been invoked often to explain the acceleration and heating of plasma particles. Solar observations suggest that hard X-rays from solar flares are produced by reconnection [3], and it has been proposed that the million degree corona is heated by turbulent reconnection [4]. In the Earth's magnetosphere, reconnection is believed to occur in the day-side magnetopause and in the magnetotail, where charged particles can be accelerated along field lines toward the polar regions, giving rise to the aurora [5]. In laboratory plasmas such as reverse-field pinches, enhanced ion heating has been seen to correlate with magnetic fluctuations associated with reconnection and dynamo activity [6]. In all these environments, however, it has not yet been possible to measure the complete reconnection magnetic field topology together with the detailed spatial and temporal characteristics of any resultant particle energization. Therefore, a rigorous demonstration of both these aspects simultaneously, which is the subject of this research, would have important implications.

Laboratory experiments have made important contributions toward understanding reconnection. In the pioneering UCLA experiments, ion acceleration, possibly reduced by wave turbulence [7], and electron heating [8] were observed. However, ion heating could not be addressed because these experiments were in the "electron MHD" regime in which $\rho_{\mathrm{i}} \gg L$. In TS-3 at the University of Tokyo, toroidal Alfvénic flows were believed to be accelerated by a "slingshot effect" of reconnected field lines, and global ion heating was attributed to thermalization of the sheared Alfvénic flows [9]. In SSX (Swarthmore Spheromak Experiment), Alfvénic ion jets correlated with reconnection were reported based on measurements of ion flux at the vacuum wall [10]. In both TS-3 and SSX, reconnection occurs when two spheromaks collide at a substantial fraction of the Alfvén speed. Thus, effects such as compressional heating or conversion of the translational energy of the spheromaks could complicate the interpretation of any observed ion acceleration and/or heating. It should be noted that in TS-3 the majority ion temperature was not measured directly but instead inferred from chord-averaged spectroscopy of neutral and impurity ion line emission, and on SSX the interpretation of retarding grid energy analyzer (RGEA) ion flux measurements 
remains an open question due to the plasma potential being unknown [11] and the inherent ambiguity in fitting a single RGEA $I-V$ characteristic to an ion distribution with both drift and thermal components.

In this work, ion heating during reconnection is studied in a controlled and wellcharacterized reconnection layer. Local ion temperature measurements are obtained rigorously using the novel, insertable Ion Dynamics Spectroscopy Probe (IDSP) [12]. The significant new result is the clear demonstration of enhanced, non-classical ion heating in a quasi 2-D, steady-state reconnection layer. It is shown that classical Ohmic heating and thermalization of ion flows are both insufficient to account for the observed ion heating, suggesting that non-classical mechanisms such as wave-particle interactions may play an important role in the energy conversion process.

Experiments were performed on MRX (Magnetic Reconnection Experiment) [13], which produces plasmas satisfying the MHD criteria globally (Lundquist number $S \gg 1, \rho_{\mathrm{i}} \ll$ $\left.L, V_{\mathrm{A}} \ll c\right)$. Formation and control of the reconnection layer in MRX is unique among laboratory experiments. "Flux-cores" containing toroidal field (TF) and poloidal field (PF) coils allow the controlled formation of a quasi 2-D, steady-state reconnection layer, shown schematically in Fig. 1. The arrows represent the motion of plasma and magnetic flux during "pull" reconnection, in which public flux linking both flux-cores is reconnected into private flux linking each individual flux-core. This sequence produces a reconnection layer elongated in $Z$ which persists for more than ten Alfvén transit times $\tau_{\mathrm{A}}$. When the TF coils are connected with opposite (same) polarity, the out-of-plane field $B_{\theta}$ is negligible (finite), resulting in null-helicity (co-helicity) reconnection. Reported results in this paper are from null-helicity experiments. Previously, the detailed structure of the reconnection layer was reported [14], revealing Y-shaped and O-shaped diffusion regions in the null-helicity and cohelicity cases, respectively, and a neutral sheet thickness $\delta \sim c / \omega_{\mathrm{pi}} \sim \rho_{\mathrm{i}}$. The reconnection rate was found to be consistent with a generalized Sweet-Parker model $[15,16]$ in which effects of compressibility, downstream pressure, and enhanced non-classical resistivity are considered. 
All three components of $\mathbf{B}$ are measured using magnetic probe arrays, and electron density $n_{\mathrm{e}}$ and temperature $T_{\mathrm{e}}$ are measured using a triple Langmuir probe. Other important reconnection quantities are inferred from the direct measurements, including the poloidal flux $\psi \equiv \int_{0}^{R} 2 \pi R^{\prime} B_{\mathrm{Z}}\left(R^{\prime}\right) d R^{\prime}$ (assuming axisymmetry), current density $j_{\theta} \approx-\left(\partial B_{\mathrm{Z}} / \partial R\right) / \mu_{0}$, reconnection electric field $E_{\theta}=-(\partial \psi / \partial t) / 2 \pi R$, plasma resistivity in the neutral layer $\eta^{*} \equiv E_{\theta} / j_{\theta}$, classical perpendicular Spitzer resistivity $\eta_{\mathrm{Sp}}$, Alfvén speed $V_{\mathrm{A}} \equiv B / \sqrt{\mu_{0} \rho}$, and even the plasma inflow speed $V_{\mathrm{R}} \approx-(\partial \psi / \partial t) /(\partial \psi / \partial R)$ (invoking flux-freezing outside the diffusion region). The present experiments use pure helium discharges in order to guarantee a direct measurement of $T_{\mathrm{i}}$ using ion Doppler spectroscopy. Parameters for the present experiments are as follows: $n_{\mathrm{e}} \approx 0.3-1 \times 10^{14} \mathrm{~cm}^{-3}, T_{\mathrm{i}} \sim T_{\mathrm{e}} \approx 5-20 \mathrm{eV}, B \approx 200-500 \mathrm{G}$, and $S \approx 250-1000$. Qualitative features of the reconnection layer for the present helium discharges are similar to those of previously reported hydrogen discharges, except $\delta_{\mathrm{He}} \approx 2 \delta_{\mathrm{H}}$, consistent with the previously reported $\delta \sim \rho_{\mathrm{i}}$ scaling [14]. Spatially, the reconnection region is located in an area given by $R \approx 35 \rightarrow 40 \mathrm{~cm}$ and $Z \approx-10 \rightarrow 10 \mathrm{~cm}$.

The major new experimental accomplishment, local measurement of $T_{\mathrm{i}}$, was made possible by the IDSP [12], which was developed at UW-Madison. The IDSP is an insertable spectroscopy probe which collects plasma light from a localized volume. Two perpendicular lines-of-sight can give simultaneous Doppler broadening and relative Doppler shift information, although only one line-of-sight is used for the present experiments. Figure 1(a) shows the placement of the IDSP in MRX (to scale); the lines-of-sight can be oriented in an $R-Z$ or $R-\theta$ plane. Further details of the probe are described elsewhere [12]. Plasma light is delivered via fiber optics to the entrance slit of a $1 \mathrm{~m}$ monochromator $(0.05 \AA$ resolution) and imaged with a CCD camera (wavelength resolution of $0.074 \AA /$ pixel). The reported $T_{\mathrm{i}}$ values are determined by fitting each measured HeII $4686 \AA$ spectrum to a single Gaussian convolved with the known instrumental broadening, a typical example of which is shown in Fig. 2. The approximately $0.3 \AA$ of fine structure in this emission line (which is slightly less than instrumental broadening) has been determined to be unimportant. Time resolution is limited by available light, which typically requires a CCD gate opening time of 
$10 \mu \mathrm{s}\left(\approx 3 \tau_{\mathrm{A}}\right)$. Stark broadening is negligible, and IDSP perturbation of the plasma was characterized carefully and seen to be minimal after 10-15 conditioning discharges. Using only one IDSP view, the relative Doppler shift could still be obtained by rotating the IDSP $180^{\circ}$ and averaging over several discharges. IDSP Doppler shifts are used to calibrate Mach probe measurements of ion flow speed derived from an unmagnetized fluid sheath theory [17] generalized for $T_{\mathrm{i}} \approx T_{\mathrm{e}}$.

Two sets of null-helicity experiments with constant helium fill pressure $(6 \mathrm{mT})$ and discharge voltage $(\mathrm{TF}=13 \mathrm{kV}$ and $\mathrm{PF}=11 \mathrm{kV})$ demonstrate unambiguously that ions are heated as a consequence of reconnection. First, the time evolution of $T_{\mathrm{i}}$ in the center of the reconnection region (IDSP light collected between $R=35 \rightarrow 40 \mathrm{~cm}$ and $Z=-2.5 \rightarrow$ $2.5 \mathrm{~cm}$; henceforth, denote this area $A$ ) is obtained for two situations: with and without reconnection. "Pull" reconnection is driven by allowing the PF current to ramp down after reaching its peak, which induces the requisite $E_{\theta}$ in the plasma from $t \approx 250 \rightarrow 280 \mu$ s. To omit reconnection, the PF circuit is shorted out (crowbarred) near peak current so that very little $E_{\theta}$ is induced. The ions are heated by more than a factor of three when reconnection is driven and much less so when it is not driven, as shown in Fig. 3. Error bars in the ordinate represent one standard deviation in an ensemble of $T_{\mathrm{i}}$ measurements (5-10 discharges), and error bars in the abscissa represent the CCD gate opening. The initial $T_{\mathrm{i}} \approx 3-6 \mathrm{eV}$ before $t=245 \mu \mathrm{s}$, common to both cases, is believed to result from dynamics of plasma formation. In the second set of experiments, the IDSP was scanned in $R$ with two CCD gate-opening times, $t=250 \rightarrow 260 \mu \mathrm{s}$ and $t=260 \rightarrow 270 \mu \mathrm{s}$, revealing a spatial dependence in the relative increase of $T_{\mathrm{i}}$, namely that the heating occurred in the vicinity of the reconnection layer, as shown in Fig. 4. The location of the reconnection layer is determined from the measured profile of the reconnecting field $B_{\mathrm{Z}}(R)$ (averaged over the same discharges), also shown in Fig. 4. The measured temporal and spatial characteristics of $T_{\mathrm{i}}$ clearly demonstrate the cause and effect of reconnection and ion heating.

In classical MHD models of reconnection [1,2], ions could be heated either in the layer through Coulomb interactions with Ohmically heated electrons or downstream from the 
layer via thermalization of the well-known Alfvénic outflow. In the present experiments, the situation is quite different. First, the ion-electron energy relaxation time is several hundred microseconds, much longer than the entire reconnection process, and therefore Coulomb interactions between ions and electrons are not relevant. Second, the sheared downstream ion flow speed $V_{\mathrm{iz}}$ during reconnection has been measured by a Mach probe to increase linearly from 0 to approximately $8 \mathrm{~km} / \mathrm{s}\left(\approx 0.25 V_{\mathrm{A}}\right)$ along the layer $(Z=0 \rightarrow 10 \mathrm{~cm}$, $R=38 \mathrm{~cm}$ ), as shown in Fig. 5. The maximum energy density of this flow is an order of magnitude smaller than the observed ion thermal energy density, implying that in this case, thermalization of the outflow cannot possibly be an important ion heating mechanism. The sub-Alfvénic $V_{\mathrm{iz}}$ in MRX is a result of high downstream pressure [15] which reduces the $\nabla p$ force along $Z$, a prime example of how global boundary conditions can affect local features of the reconnection layer. Even if higher energy flows were to develop downstream, they could not easily explain the ion heating measured by the IDSP at $Z=0 \mathrm{~cm}$.

An ion energy budget for the reconnection process can be determined. The amount of energy released due to reconnection can be understood through Poynting's theorem, $\boldsymbol{-} \boldsymbol{\nabla} \cdot(\mathbf{E} \times \mathbf{B}) / \mu_{0}=\partial\left(B^{2} / 2 \mu_{0}\right) / \partial t+\mathbf{E} \cdot \mathbf{j}$. The LHS is the Poynting flux, which physically represents the incoming magnetic energy density per unit time and the work done per unit volume by the Maxwell stress tensor. The first term on the RHS is the time rate of change of magnetic field energy density, and the second term is the power dissipation per unit volume due to reconnection. In a steady-state situation, which is well approximated in MRX, $\mathbf{E} \cdot \mathbf{j}\left(\approx E_{\theta} j_{\theta}\right)$ is balanced by the Poynting flux. Therefore the total field energy released due to reconnection $W_{\text {rec }}$ from $t_{1}=245 \mu \mathrm{s} \rightarrow t_{2}=265 \mu$ s in volume $V$ (the area $A$ revolved around the axis of symmetry) can be calculated easily: $W_{\text {rec }} \equiv \int_{V}\left(\int_{t_{1}}^{t_{2}} E_{\theta}(t) j_{\theta}(t) d t\right) d^{3} V \approx 5 \mathrm{~J}$ for the discharge conditions pertaining to Figs. 3 and 4. Easily more than half of this energy $(>3 \mathrm{~J})$ is converted to ion kinetic energy $W_{\text {ions }}$, which includes (1) an increase in ion thermal energy $\Delta W_{\mathrm{th}, \mathrm{i}} \equiv(3 / 2) \int_{V}\left[n\left(t_{2}\right) T_{\mathrm{i}}\left(t_{2}\right)-n\left(t_{1}\right) T_{\mathrm{i}}\left(t_{1}\right)\right] d^{3} V \approx 0.5 \mathrm{~J},(2)$ directed flow energy $W_{\text {outflow }} \equiv \int_{V}\left(\rho V_{\mathrm{i}}^{2} / 2\right) d^{3} V \approx 0.1 \mathrm{~J},(3)$ ion heat loss due to convection $W_{\text {convection }} \approx 1 \mathrm{~J}$, and (4) estimated ion heat loss due to classical conduction $W_{\text {conduction }} \gtrsim 1.4 \mathrm{~J}$. Ion energy loss to 
neutrals, predominantly via charge-exchange, is estimated to be negligible. The remainder of $W_{\text {rec }}$ goes to the electrons. $W_{\text {rec }}$ is about ten times larger than the expected energy release due to classical Ohmic dissipation, i.e. $E_{\theta} j_{\theta} \approx 10 \eta_{\mathrm{Sp}} j_{\theta}^{2}$ (estimated $Z_{\mathrm{eff}} \lesssim 1.2$ ). From the energy budget, it is clear that the dominant dissipation mechanism responsible for the ion heating must be a non-classical process.

The relative importance of non-classical versus classical dissipation is embodied in the ratio $\eta^{*} / \eta_{\text {Sp }}$, which was shown previously to scale with collisionality [15]. By varying the discharge voltage, $\eta^{*} / \eta_{\mathrm{Sp}}, W_{\mathrm{rec}}$, and $\Delta W_{\mathrm{th}, \mathrm{i}}$ are varied. The correlation between $\Delta W_{\mathrm{th}, \mathrm{i}}$ and $W_{\text {rec }}$ is quite strong as shown in Fig. 6(a), further supporting the claim that reconnection is indeed heating the ions. More suggestive, however, is the fact that the fraction $\Delta W_{\mathrm{th}, \mathrm{i}} / W_{\text {rec }}$ increases from approximately $4 \%$ to $14 \%$ as $\eta^{*} / \eta_{\text {Sp }}$ increases from 8 to 15 , as shown in Fig. 6(b). (Note that $\Delta W_{\mathrm{th}, \mathrm{i}}$ is only the remnant ion thermal energy in volume $V$ minus the losses due to convection and conduction, but the trend is the important point here.) A possible explanation is that as the plasma becomes more collisionless, turbulence can scatter the current-carrying particles, increasing $\eta^{*}$ and also heating the ions more effectively. The subtle hint provided by Fig. 6(b) is that whatever (non-classical) mechanism is determining the enhanced reconnection rate (and hence increased reconnected field energy) is also responsible for channeling that reconnected field energy to the ions. This effect should appear in any mechanism proposed to explain the enhanced $\eta^{*} / \eta_{\text {Sp }}$ observed in MRX.

To summarize, local ion heating due to reconnection has been demonstrated definitively using the novel IDSP diagnostic. This is a significant result because it has implications for many solar and space physics phenomena in which reconnection is invoked heuristically to explain the presence of energetic particles. It is estimated that more than half of the reconnected field energy is converted to ion kinetic energy in MRX. The ion heating is attributed to non-classical dissipation mechanisms because thermalization of the measured sub-Alfvénic ion outflow and the energy available from classical Ohmic dissipation are both insufficient to account for the observed energy conversion to ions. The ion heating characteristics suggest that non-classical mechanisms such as wave-particle interactions could play an important 
role not only in determining the reconnection rate but also in heating the ions.

In many aspects, the reconnection scenario presented here is fundamentally different from classical MHD reconnection models, which feature Ohmic heating and Alfvénic jets. It is worth noting that the present "quiet-heating" scenario with no energetic flows, which would be difficult to detect in the solar corona, could play a role in coronal heating. It is also worth noting that the absence of Alfvénic flows in MRX is understood to be a consequence of the high downstream pressure, suggesting that global boundary conditions should in general affect local reconnection dynamics. Theoretically, it is possible for current-driven instabilities to produce the observed effects presented in this paper, and experimentally, efforts are underway to measure fluctuations in hopes of identifying candidate modes. A laser-induced fluorescence system under development will allow further studies of ion heating, including non-isotropic effects, with much improved space and time resolution.

The authors thank D. Cylinder for technical assistance and Drs. F. Trintchouk, F. Levinton, and R. Bell for helpful discussions regarding the spectroscopy. S.C.H. and T.A.C. acknowledge the support of NASA-GSRP fellowships. MRX is funded by NSF, NASA, and DOE.

*Department of Physics, University of Wisconsin-Madison, 1150 University Ave., Madison, WI 53706 


\section{REFERENCES}

[1] V. M. Vasyliunas, Rev. Geophys. Space Phys. 13, 303 (1975)

[2] D. Biskamp, Physics Reports 237, 179 (1994).

[3] S. Masuda et al., Nature 371, 495 (1994).

[4] E. R. Priest et al., Nature 393, 545 (1998).

[5] J. W. Dungey, Phys. Rev. Lett. 6, 47 (1961).

[6] e.g. E. Scime et al., Phys. Fluids B 4, 4062 (1992).

[7] W. Gekelman, R. L. Stenzel, and N. Wild, J. Geophys. Res. 87, 101 (1982).

[8] R. L. Stenzel, W. Gekelman, and N. Wild, J. Geophys. Res. 87, 111 (1982).

[9] Y. Ono et al., Phys. Rev. Lett. 76, 3328 (1996).

[10] T. W. Kornack, P. K. Sollins, and M. R. Brown, Phys. Rev. E 58, R36 (1998).

[11] T. W. Kornack, private communication (1999).

[12] G. Fiksel, D. J. D. Hartog, and P. W. Fontana, Rev. Sci. Instr. 69, 2024 (1998).

[13] M. Yamada et al., Phys. Plasmas 4, 1936 (1997).

[14] M. Yamada et al., Phys. Rev. Lett. 78, 3117 (1997).

[15] H. Ji, M. Yamada, S. Hsu, and R. Kulsrud, Phys. Rev. Lett. 80, 3256 (1998).

[16] H. Ji et al., Phys. Plasmas 6, 1743 (1999).

[17] M. Hudis and L. M. Lidsky, J. App. Phys. 41, 5011 (1970). 


\section{FIGURES}

(a)

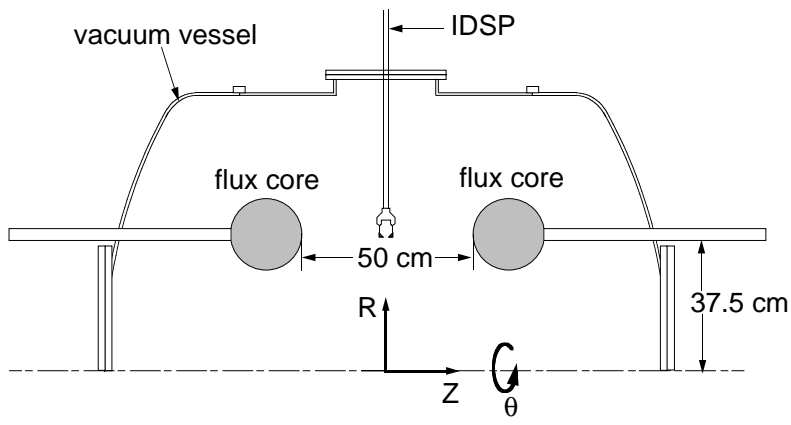

(b)

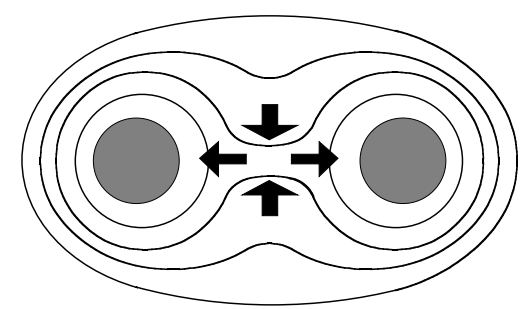

FIG. 1. (a) Schematic of MRX upper half plane, showing placement of flux-cores and IDSP. (b) Illustration of "pull" reconnection in which "public" poloidal flux reconnects into "private" poloidal flux.

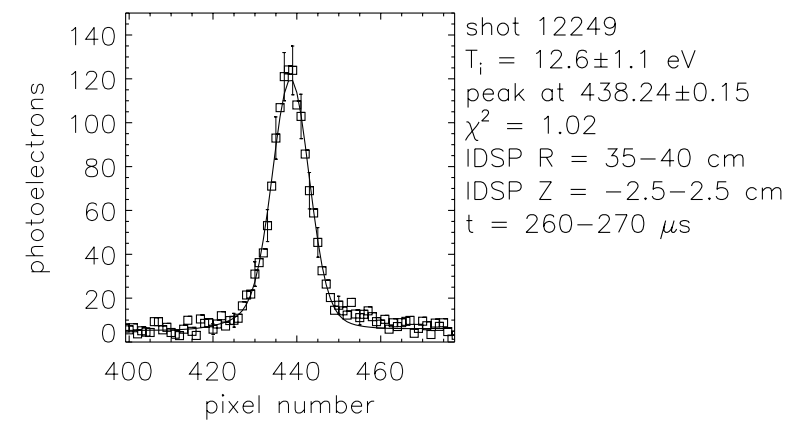

FIG. 2. Doppler broadened profile of HeII $4686 \AA$ fitted by the convolution of a Gaussian and instrumental broadening. 


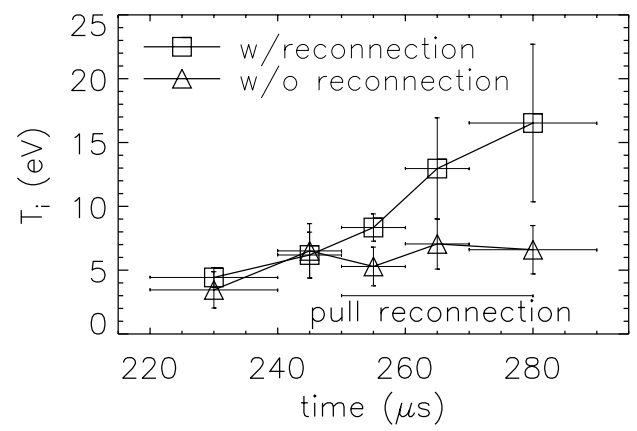

FIG. 3. Time evolution of $T_{\mathrm{i}}$ in the reconnection region with and without reconnection, showing the clear effect of reconnection on ion heating.

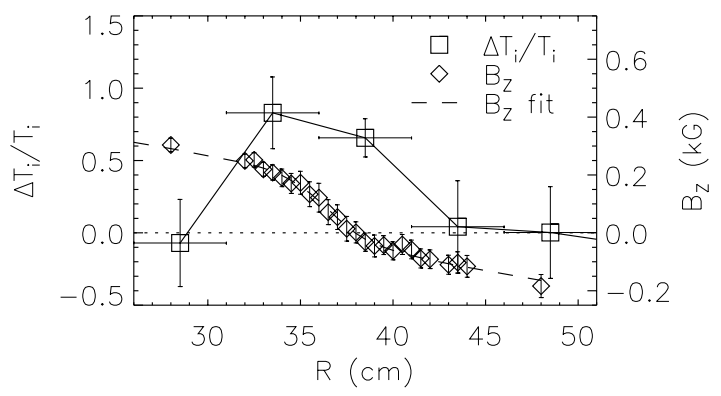

FIG. 4. Radial profiles of the relative rise of $T_{\mathrm{i}}$ from $250-270 \mu$ s and reconnecting field $B_{\mathrm{Z}}$ averaged over the same time. Substantial heating occurs in the reconnection layer (between the knees of the $B_{\mathrm{Z}}$ profile).

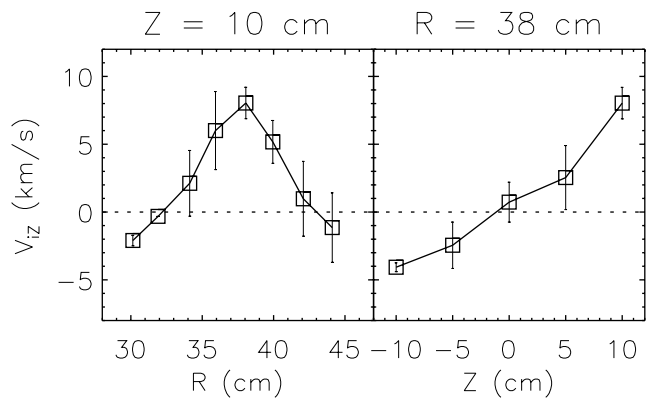

FIG. 5. Downstream ion drift speed $V_{\text {iZ }}$ versus $R$ at $Z=10 \mathrm{~cm}$ (left) and versus $Z$ at $R=38 \mathrm{~cm}$ (right) averaged over $t=250-270 \mu \mathrm{s}$. (Upstream $V_{\mathrm{A}} \approx 35 \mathrm{~km} / \mathrm{s}$.) 

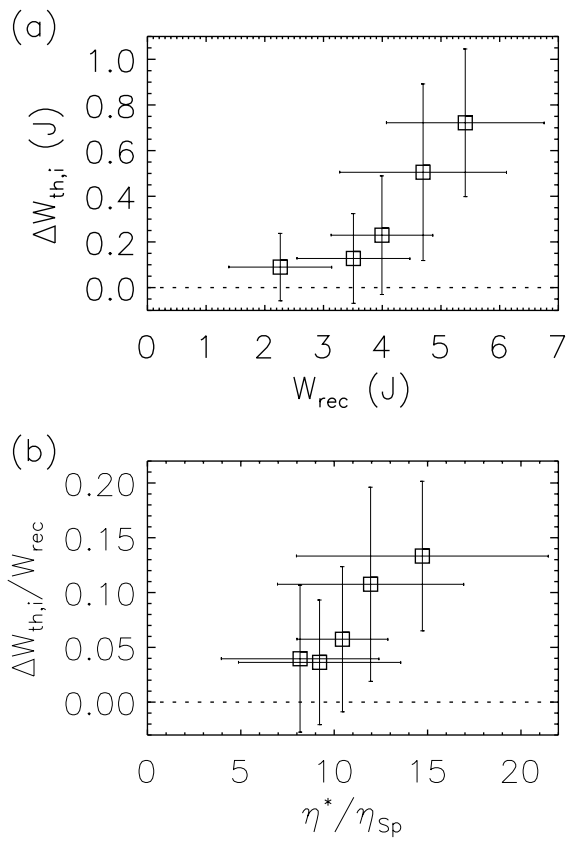

FIG. 6. Discharge voltage scan at constant helium fill pressure (6 $\mathrm{mT})$. (a) Increase in ion thermal energy $W_{\mathrm{th}, \mathrm{i}}$ versus reconnected magnetic field energy $W_{\text {rec }}$ from $t=245 \rightarrow 265 \mu \mathrm{s}$ in the reconnection layer. (b) Increase in $W_{\mathrm{th}, \mathrm{i}} / W_{\text {rec }}$ versus $\eta^{*} / \eta_{\mathrm{Sp}}$. 